\title{
BMJ Open Face-to-face physiotherapy compared with a supported home exercise programme for the management of musculoskeletal conditions: protocol of a multicentre, randomised controlled trial - the REFORM trial
}

Hannah G Withers (D) , Joanne V Glinsky, ${ }^{1,2}$ Jackie Chu, ${ }^{1}$ Matthew D Jennings, ${ }^{3}$ Alison J Hayes, ${ }^{4}$ lan J Starkey, ${ }^{5}$ Blake A Palmer, ${ }^{5}$ Lukas Szymanek, ${ }^{6}$ Jackson J Cruwys, ${ }^{6}$ David Wong, ${ }^{7}$ Kitty Duong, ${ }^{7}$ Anne Barnett, ${ }^{8}$ Matthew J Tindall, ${ }^{8}$ Barbara R Lucas, ${ }^{9}$ Tara E Lambert, ${ }^{9}$ Catherine Sherrington (i) , ${ }^{10}$ Christopher G Maher, ${ }^{11}$ Manuela L Ferreira, ${ }^{12}$ Deborah A Taylor, ${ }^{9}$ Lisa A Harvey ${ }^{1}$

To cite: Withers HG, Glinsky JV, Chu J, et al. Face-to-face physiotherapy compared with a supported home exercise programme for the management of musculoskeletal conditions: protocol of a multicentre, randomised controlled trialthe REFORM trial. BMJ Open 2021;11:e041242. doi:10.1136/ bmjopen-2020-041242

\section{- Prepublication history and} additional supplemental material for this paper are available online. To view these files, please visit the journal online (http://dx.doi.org/10.1136/ bmjopen-2020-041242).

Received 04 June 2020 Revised 24 February 2021 Accepted 12 April 2021

Check for updates

(c) Author(s) (or their employer(s)) 2021. Re-use permitted under CC BY-NC. No commercial re-use. See rights and permissions. Published by BMJ.

For numbered affiliations see end of article.

Correspondence to Professor Lisa A Harvey; I.harvey@usyd.edu.au

\section{ABSTRACT}

Introduction Exercise, support and advice are considered core components of management for most musculoskeletal conditions and are typically provided by physiotherapists through regular face-to-face treatments. However, exercise can be provided remotely as part of a home exercise programme, while support and advice can be provided over the telephone. There is initial evidence from trials and systematic reviews to suggest that remotely provided physiotherapy can be used to manage a variety of musculoskeletal conditions safely and effectively. Methods and analysis The aim of this single-blind randomised controlled non-inferiority trial is to determine whether a supported home exercise programme is as good as or better than face-to-face physiotherapy for the treatment of musculoskeletal conditions. Two hundred and ten participants will be recruited from five public hospitals in Sydney, Australia. Participants will be randomised to either the supported home exercise group or the faceto-face physiotherapy group. Participants allocated to the supported home exercise group will initially receive one face-to-face session with the trial physiotherapist and will then be managed remotely for the next 6 weeks. Participants allocated to the face-to-face physiotherapy group will receive a course of physiotherapy as typically provided in Sydney government hospitals. The primary outcome is function measured by the Patient Specific Functional Scale at 6 weeks. There will be nine secondary outcomes measured at 6 and 26 weeks. Separate analyses will be conducted on each outcome, and all analyses will be conducted on an intention-to-treat basis. A health economic evaluation will be conducted from a health funder plus patient perspective.

Ethics and dissemination Ethical approval was obtained on the 17 March 2017 from the Northern Sydney Local Health District HREC, trial number HREC/16HAWKE/431RESP/16/287. The results of this study will be submitted for publication to peer-reviewed journals and be presented

\section{Strengths and limitations of this study}

The intervention that is being investigated minimises reliance on face-to-face treatments and as such is highly relevant to the current COVID-19 pandemic.

- The trial has many design features important for minimising bias including concealed allocation, blinded assessors and intention-to-treat analysis.

- This trial is highly pragmatic involving five public hospitals in Sydney, which increases its external validity.

- Although the 6-week and 26-week assessments are blinded, it is not possible to blind the clinicians or the participants.

- The results of this trial will be most applicable to the provision of physiotherapy in public hospitals as no participants from the private physiotherapy sector will be included.

at national and international conferences. Recruitment commenced in March 2019, and it is anticipated that the trial will be completed by December 2021. This trial will investigate two different models of physiotherapy care for people with musculoskeletal conditions.

Trial registration number $\mathrm{CPMP/ICH}-135 / 95$.

Protocol version The most recent version of the protocol is V.1.2 dated November 2019.

\section{INTRODUCTION}

Musculoskeletal conditions are common and include back pain, hip and knee osteoarthritis, whiplash-associated disorders and ankle sprains. Together musculoskeletal conditions cause $21 \%$ of the total years lived with disability (second only to mental illness), 
placing a great burden on world health. ${ }^{1}$ In 2015 , an estimated $30 \%$ of all people had at least one musculoskeletal condition in Australia. ${ }^{2}$ This figure is reported to be as high as $72 \%$ for people aged over 75 years. ${ }^{3}$ In 2008-2009, costs attributed to musculoskeletal conditions were an estimated $\$ 5.7$ billion. ${ }^{45}$

Exercise, support and advice are considered core components of management for many musculoskeletal conditions. ${ }^{6-9}$ Exercise, support and advice are typically provided by physiotherapists through regular face-to-face treatments. However, exercise can be provided remotely as part of a home exercise programme while support and advice can be provided via the telephone. There is initial evidence from trials and systematic reviews to suggest that different forms of remotely provided physiotherapy can be used to manage a variety of musculoskeletal conditions safely and effectively. ${ }^{6} 10-18$ A move away from reliance on face-to-face physiotherapy has many potential benefits. Adopting new technologies and strategies into physiotherapy management will allow for the delivery of timely and accessible care to those who are in remote or rural locations and those who have significant mobility issues. Another benefit for this method of physiotherapy is its low cost, which might enhance cost-effectiveness from a funder and patient perspective. Increasing remote access and decreasing the cost of physiotherapy may have the added benefit of decreasing the burden on the public health system by decreasing waiting times for publicly funded outpatient physiotherapy. ${ }^{19}$

This model of care is particularly relevant given the global COVID-19 pandemic, although it was developed prepandemic. In Sydney, Australia, and elsewhere, the pandemic has meant that telerehabilitation strategies have been rapidly adopted by many hospital outpatient clinics. This has allowed physiotherapists to support the social isolation policies in place to reduce the spread of COVID-19. Telerehabilitation has enabled physiotherapists to continue to provide services to some of the many patients requiring physiotherapy thereby potentially preventing the escalation of symptoms and presentation to emergency departments at a time of burden for the health system. ${ }^{20}$

The trial will be highly pragmatic with broad inclusion criteria to capture a range of musculoskeletal conditions for which exercise, support and advice are the basis of evidence-based care. The aim is to determine whether a supported home exercise programme is as effective or better than a course of face-to-face physiotherapy. This will be determined with one primary outcome and nine secondary outcomes. An economic analysis will be run alongside the trial to assess the affordability and value for money of this model of care from a health funder plus patient perspective. A process evaluation will also be completed in order to understand the feasibility of delivering physiotherapy through supported home exercise programmes and to explore the perspectives of patients, healthcare professionals and key stakeholders about different models of delivering physiotherapy.

\section{METHODS AND ANALYSIS}

\section{Design}

A single-blind randomised controlled non-inferiority trial will be undertaken to compare a course of physiotherapy as typically provided in Sydney government hospitals with a supported home exercise programme administered through a smartphone/tablet application (an 'app') and supplemented with text messages and two telephone calls. Cost-effectiveness will be evaluated from a health funder and patient perspective.

Participants will be recruited from five tertiary public teaching hospitals in Sydney, Australia: Bankstown Lidcombe Hospital, Blacktown-Mt Druitt Hospital, Campbelltown Hospital, Hornsby Hospital and Liverpool Hospital.

\section{Participants}

Two hundred and ten adults with a musculoskeletal condition presenting for a course of physiotherapy or on a waiting list for physiotherapy at one of the five participating hospitals will be recruited.

A person will be eligible to participate if he or she:

- Is 18 years or over and able to provide informed consent in writing.

- Has a musculoskeletal condition. Examples include:

- Back/neck pain.

- Hip or knee osteoarthritis.

- Whiplash-associated disorders.

- Ankle sprains.

- Postfracture.

- Sporting injury.

- Post hip or knee replacement.

- Is seeking physiotherapy treatment at the participating hospital.

- Can speak and read English to provide informed consent.

- Is able to participate for 6 weeks and will be available for 6-week and 26-week follow-up assessments.

- Has access to a smartphone with internet connection.

- Is identified by the hospital physiotherapists or trial physiotherapist (study coordinator) to have a condition appropriate for treatment with exercise, support and advice.

A person will be excluded if he or she:

- Is pregnant.

- Has a mental illness that may affect adherence to the trial protocol. This will be determined in consultation with the treating physiotherapists and a review of medical history.

- Is deemed to be at a high risk of falling with home exercises.

- Is at a clinical risk without face-to-face physiotherapy.

- Is on a postoperative exercise regimen prescribed by a surgeon.

\section{Public and patient involvement}

Over a 20-year period, patients and the public were involved in the development of the exercise app (www. 
physiotherapyexercises.com) on which this trial is based. The primary outcome measure was developed in $1995^{18}$ with input from patients. All participants for this trial are patients on a waiting list for outpatient physiotherapy in one of the five public hospitals involved in this trial. All participants will be asked to give written informed consent before being randomised. In order to include the participants' perspective in the results of this trial, an outcome measure asking the participants to self-report their satisfaction with service delivery will be included. A secondary process evaluation will also explore participants' opinions and experiences of the intervention and trial. A separate manuscript is being prepared to explain the protocol for the process evaluation. Participants will be able to access the published results of this trial.

\section{Recruitment strategy and time frame}

Recruitment started in March 2019, and currently, 141 participants have been randomised. Recruitment was however temporarily ceased on 9 March 2020 because of the COVID-19 pandemic. It recommenced gradually from July 2020, once it was considered safe and appropriate by the investigators and participating sites and will continue until 210 participants have been recruited (see online supplemental appendix table 1 for the timeline of study).

Potential participants will be screened according to the inclusion/exclusion criteria from the waiting list of each outpatient physiotherapy department. This process will be completed by either the treating physiotherapists or administrative staff of the department over the telephone. If appropriate, patients will be given an appointment to attend the outpatient department to complete the consent, baseline assessment and randomisation.

\section{Assignment of intervention}

A secure random allocation schedule has been computer generated by an independent researcher and is stored off site on a REDcap database. Randomisation is blocked and stratified by site and duration since onset of injury (less than 12 weeks vs more than 12 weeks). The allocation schedule is concealed from potential participants and from all staff associated with the trial. Randomisation will occur once a participant has been screened, provided consent and completed the baseline assessment. A trial staff member responsible for coordinating the treatments will $\log$ onto REDcap to retrieve the participant's allocation. Participants' assignments will not be disclosed to the blinded assessors or all but two investigators. Eligible participants are randomised into one of two groups namely:

\section{The supported home exercise group}

Participants initially receive one face-to-face session with the trial physiotherapist but are then managed remotely for the next 6 weeks.
The face-to-face physiotherapy group

Participants receive a course of face-to-face physiotherapy by a hospital physiotherapist.

\section{Interventions}

Supported home exercise group

Participants allocated to the supported home exercise group initially receive one face-to-face session with the trial physiotherapist and then will be managed remotely for the next 6 weeks. During the initial session, the trial physiotherapist will assess the patient and then prescribe an individualised 6-week home exercise programme consisting of a battery of 5-10 exercises. This will be delivered to patients' mobile devices using a freely available exercise-prescribing app that authors LAH, JVG and colleagues have developed (www.phys iotherapyexercises.com). The number of repetitions and sets of exercises will be determined by the trial physiotherapist. Participants will be asked to complete their exercises at least once every day for the intervention period of 6 weeks. Participants will record exercise adherence on their app. These data will be automatically transferred to a password-protected section of the website, which is accessed by the trial physiotherapist to remotely monitor exercise adherence. The trial physiotherapist will provide ongoing support through weekly text messages. The purpose of these text messages is to encourage adherence to the prescribed exercises and provide the participants with encouragement and support. These text messages are generated from a prepaid website and are scheduled to be sent each week to the participants in the supported home exercise group. The messages are not individualised but are designed to be motivating and to remind participants to continue their exercises. Participants cannot respond to these text messages (see online supplemental appendix table 2 for examples of the text messages). The participants will also receive a telephone call from the trial physiotherapist at 2 and 4 weeks to ensure adherence and provide feedback, support and advice. Participants will be telephoned more frequently if their exercise adherence is poor. Participants are also able to contact the trial physiotherapist on a study mobile phone number or via email at any time. The trial physiotherapist has the option of providing an additional face-to-face physiotherapy session if she has any concerns about a participant's progress, safety or well-being that she may become aware of from conversations with the participant over the telephone or from any other trial or hospital staff. Details about all additional text and phone calls with the intervention participants will be recorded including the number of text messages and the number and duration of telephone calls. In addition, the number of failed attempts to contact participants by telephone will be recorded. Detailed notes will also be kept regarding participants' adherence to their exercise programmes and any advice and support given. Participants will also be asked to report on whether or not they received the weekly autogenerated text messages.

\section{Face-to-face physiotherapy group}

Participants allocated to the face-to-face physiotherapy group will receive a course of physiotherapy as typically provided 
in Sydney government hospitals. This will be provided by the hospital physiotherapists and could involve up to three sessions per week for up to 6 weeks or group classes. The number of sessions per week and duration of the course of physiotherapy for each participant will be determined by the hospital physiotherapist and may be gradually decreased and completed during the intervention period if a participant recovers. This approach has been adopted to mimic usual practice. The type of physiotherapy provided during the face-to-face sessions will be determined by the hospital physiotherapist and may include any combination of manual therapy, advice, exercise and occasional electrotherapy. In this way, the trial will be pragmatic and will provide a reallife comparison of the two models of care. The number of sessions and type of therapy provided will be recorded and reported (see online supplemental appendix table 3 for a detailed description of the intervention as per the Template for Intervention Description and Replicaton (TIDieR) checklist).

Participants in both groups are permitted to continue with any concomitant treatments for any comorbidities. Participants in both groups will be asked not to pursue other sources of physiotherapy for their current musculoskeletal conditions over the 6 -week intervention period.

\section{Outcome measures}

All outcomes will be collected at baseline, 6 weeks and 26 weeks except one outcome (participant satisfaction with healthcare service delivery), which will only be collected at 6 and 26 weeks (see online supplemental appendix table 4 for the trial visit schedule). Site, duration since onset of injury (less than 12 weeks vs more than 12 weeks) and baseline measurements will be used as covariates in the analyses to increase the precision of the estimates.

The primary outcome will be:

\section{Function as measured by the patient-specific functional scale at 6} weeks

This outcome measure is sensitive to changes that are important to patients and is used across many different types of musculoskeletal conditions including cervical spine, knee and lower back pain. ${ }^{18}$ Participants are asked at baseline to identify up to five functional activities that are most important to them and which they find difficult to perform. Participants are then asked to rate each activity at baseline and 6 weeks on an 11-point scale. The scale ranges from 0 to 10 and indicates the level of difficulty participants have with each activity due to their condition. Zero indicates that they are unable to perform the activity and 10 indicates that they are able to perform the activity at preinjury level. Scores for each activity are summed and expressed as a percentage of the total possible score for the participant (determined by the number of identified activities)..$^{1821}$

The secondary outcomes will be:

The patient-specific functional scale at 26 weeks

See previous information for details.
Fear of movement and reinjury measured using the Tampa Scale for Kinesiophobia (TSK) at 6 and 26 weeks

The TSK is a multi-item instrument that quantifies fear of movement and reinjury. Participants are asked to score 17 items on a scale of $1-4$, where a score of 1 indicates "strongly disagree' and a score of 4 indicates 'strongly agree'. Items 4,8 , 12 and 16 are reversed where 1 indicates 'strongly agree' and 4 indicates 'strongly disagree'. This instrument has high reliability. $^{22} 23$

Pain measured using a 0-10 Numerical Rating Scale (NRS) at 6 and 26 weeks

Participants are asked to rate their average pain over the past 24 hours on a $0-10$ NRS anchored at each end with 'no pain' and 'worst pain imaginable'. The NRS for pain measurement is a valid and reliable tool for measuring acute and chronic pain. ${ }^{24}$

\section{Patient global impression of change at 6 and 26 weeks}

Participants are asked to rate the change in their condition on a numerical scale. This scale ranges from negative seven to positive seven anchored in the middle and at each end with 'no change', 'very much worse' and 'very much better', respectively.

Patient satisfaction with healthcare service delivery at 6 weeks Participants are asked to rate their satisfaction with the care they have received for their musculoskeletal condition on an 11-point numerical scale. This scale ranges from 0 to 10 anchored at each end with 'complete dissatisfaction' and 'complete satisfaction' with the delivery of healthcare service.

Health-related quality of life measured using the EuroQol-5D at 6 and 26 weeks

This validated questionnaire has been used in a wide range of musculoskeletal conditions and requires the participant to rate their level of problems in five dimensions including mobility, self-care, usual activities, pain and anxiety/depression. Utility-based quality of life will be derived from the Australian valuation of this instrument for use in the cost-utility analysis.

Functional performance measured with the function component of the later life function and disability instrument at 6 and 26 weeks This standardised 32-item instrument captures participants' perceptions about their abilities to perform discrete actions or activities (eg, unscrew the lid of a jar and put on and take off a coat or jacket). It is suitable for adults of all ages even though it was specifically designed for adults in later life. This instrument has good validity and has been recommended for self-reported data collection. ${ }^{25}$ The full assessment also captures life performing tasks and limitations on performing life performance tasks but only the functional performance aspect of the assessment will be used. Participants are asked to rate their difficulties performing each of the 32 actions or activities on a five-point scale ranging from 'none' (ie, no difficulties performing the activity) to 'can't do'. Scores will be 
transformed into a 0-100 summary score where a high score indicates a higher level of functioning. ${ }^{26}$

Frequency of performing life tasks measured with the disability component of the later life function and disability instrument at 6 and 26 weeks

This standardised 16-item instrument captures participants' perceptions about the frequency with which they perform socially defined life tasks such as visiting friends and family in their homes, taking part in recreational activities and travelling with overnight stays. ${ }^{25}{ }^{26}$ Participants are asked 'to what extent they feel limited in doing a particular task'. They are provided with the following options: 'completely', 'a lot', 'somewhat', 'a little' and 'not at all. Scores will be transformed into a 0-100 summary score where a high score indicates a higher level of functioning. ${ }^{26}$

Limitations in capability of performing life tasks measured with the disability component of the later life function and disability instrument at 6 and 26 weeks

This standardised 16-item instrument captures participants' perceptions about their limitations in performing socially defined life tasks such as visiting friends and family in their homes, taking part in recreational activities and travelling with overnight stays. ${ }^{25}$ Participants are asked 'how often do they do a particular task'. They are provided with the following options: 'very often', 'often', 'once in a while', 'almost never' and 'never'. Scores will be transformed into a $0-100$ summary score where a high score indicates a higher level of functioning. ${ }^{26}$

\section{Sample size}

A sample size of 210 people is required to provide $80 \%$ power for a non-inferiority margin (delta) of -1.5 points on the primary outcome Patient Specific Functional Scale (PSFS) where a positive between-group difference favours the supported home exercise group assuming a 15\% loss to follow-up, an SD of $2,{ }^{21}$ a $15 \%$ treatment dropout rate, a correlation between baseline and final scores of 0.5 and a conservative estimate that the between-group difference favours the face-to-face group by 0.75 points.

\section{Data analysis}

Statistical plan

Data analysis and dissemination of results will occur after the database has been cleaned and locked. All analyses will be conducted on an intention-to-treat basis with these performed and interpreted blinded to treatment group according to a prespecified statistical analysis plan. Separate analyses will be conducted on each outcome.

\section{Non-inferiority analysis}

The supported home exercise group will be considered non-inferior to the face-to-face physiotherapy group if the upper limit of the $95 \%$ CI associated with the mean between group difference on the PSFS at 6 weeks indicates that supported home exercise versus face-to-face physiotherapy is either better or no worse than 1.5 points out of 10 . The non-inferiority cut-off point of 1.5 was decided by the investigators after taking into consideration the likely implications of this amount of difference on function and the cost of the intervention.

\section{Other analyses}

The results of all other analyses will be presented as point estimates (with 95\% CI) and will not be interpreted with respect to non-inferiority margins (deltas) or statistical significance but instead used to aid the interpretation of the results of the non-inferiority analysis of the primary outcome at 6 weeks. We will not make any adjustments for multiple comparisons; however, we will interpret these findings cautiously taking into account the number of outcomes and the two endpoints. Between-group comparisons of each outcome will be conducted using regression models in which the outcome will be a linear function of a dummy-coded variable representing group membership (supported home exercise group or face-to-face physiotherapy group) and a dummy-coded variable for stratum, specifically site and duration since onset of injury (less than 12 weeks vs more than 12 weeks). Baseline scores will be included in the model to increase statistical precision. If more than $5 \%$ of data are missing for a particular analysis, multiple imputation will be used to account for missing data provided the missing at random assumption appears plausible.

\section{Economic evaluation}

The economic evaluation will compare the supported home exercise programme with face-to-face physiotherapy and will be conducted from a health funder plus patient perspective, since patients will contribute time and money to the treatments. If supported home exercise is statistically non-inferior to face-to-face physiotherapy, then a cost minimisation analysis will be conducted; otherwise, a cost-effectiveness analysis for the primary and secondary outcomes (patient function at 6 weeks and 26 weeks) will be conducted. A trial-based cost-utility analysis for quality of life outcomes at 26 weeks will also be conducted. The cost of delivering the physiotherapy intervention in the two arms of the trial will be determined using standard microcosting methods. All costs will be collected during the trial period and valued in 2021 Australian dollars. Health funder costs will include physiotherapists' time and materials where appropriate. Other healthcare utilisation (eg, visits to doctors, exercise physiologists and masseurs) will be determined by patient self-report. Patient costs will include the costs associated with the time to: attend the face-to-face sessions with the physiotherapist (including travel time), receive the telephone calls from the trial physiotherapist and to complete the prescribed home exercise programme. The cost of any equipment purchased will also be included. As in all economic evaluations, the costs captured in this study are likely to be skewed, so nonparametric bootstrap methods will be used for hypothesis testing and interval estimation. In the cost-utility analysis, patient outcomes will be measured in quality-adjusted life years (QALYs) 
at 26 weeks, using a standard instrument, the Euro-Quol quality of life questionnaire, the EQ-5D-5L. The incremental cost-effectiveness ratio (ICER) will be determined in Australian Dollars (AUD) per QALY gained. Bootstrapped cost-effect pairs will be plotted on an incremental cost-effectiveness plane and a cost-effectiveness acceptability curve will be generated for the probability of being cost-effective at different thresholds. The robustness of the ICERs will be tested through multiple one-way sensitivity analyses.

\section{Data collection}

Baseline data will be collected on paper case report forms (CRFs) and then entered into an electronic database (REDcap) by the trial physiotherapist. The data at 6 and 26 weeks will be collected in one of four ways. Most participants will be guided while they use an online data collection form or the assessor will take responses from participants over the telephone and enter them into the online data collection form for the participant. If the participant prefers a paper copy to be sent in the mail, then the assessor will take responses from the participant over the telephone and enter them into the database while the participants read the questions from the paper copy. Participants will also be given the option to complete the assessment on paper and return the completed forms via an included prepaid envelope. The final option of data collection will allow the participant to complete the online assessment independently by receiving a link via email and completing the questions online without any assistance from the assessor. Regardless of the method used to collect the data, the assessor responsible for interacting with the participant and/or collecting the data over the telephone will be blinded to the treatment. In addition, participants will be reminded at the time of the assessment not to reveal any details regarding their physiotherapy treatments to the assessor. If unblinding occurs, a new blinded assessor will complete the next assessment for that participant. Data for the economic evaluation will be collected over the telephone by an unblinded trial physiotherapist after the 6-week blinded assessment has been completed.

\section{Data storage}

All information collected for this trial will have identifying information removed and will be kept confidential and secure. All files containing participants' personal details will remain at the site where they are collected. The original CRFs will be stored centrally on completion of the trial and will only contain the participants' ID code. Electronically transcribed data will be stored on the REDcap system managed by the University of Sydney. Access to data will only be granted to the principal investigators and other research staff directly involved in the study. All source documents and trial documentation will be kept in a secure location by the investigators for 15 years or the appropriate retention period according to local regulations.

\section{Data confidentiality}

Consent forms, baseline assessments and all files containing participants' personal details will remain at the site where the participant was recruited. Compulsory medical notes will be completed on the electronic medical record system used in public hospitals in Sydney, Australia. All other data, both paper and electronic, will be stored either centrally in a secure location or in the password-protected database managed by the University of Sydney. All data will be deidentified.

\section{Trial monitoring}

The study will be overseen and monitored by the research staff who will examine study procedures, ensure data quality and monitor compliance with the study protocol. All protocol violations will also be recorded. An independent Data Safety Monitoring Board will not be used for this trial, and an interim analysis will not be conducted because the intervention is unlikely to cause harm, and the trial is not sufficiently large enough to warrant stopping it early on the grounds of futility. Ethical approval was obtained on the 17 March 2017 from the Northern Sydney Local Health District HREC, trial number HREC/16HAWKE/431-RESP/16/287.

All serious adverse events (SAEs) from the time of randomisation to the 26-week assessment will be recorded. These will include any events that result in death, disability, hospitalisation or prolongs existing hospitalisation. The trial physiotherapist will record all the relevant information regarding each SAE including the type of event, the start and stop dates, the action taken and the cause of the event. ${ }^{27}$ It will be reported to the principal investigator within 24 hours and reported immediately to the ethics committee irrespective of group allocation. It will also be detailed in the annual report. ${ }^{28}$ If a SAE has a significant safety issue, a report will be made to the principal investigator within 72 hours, and the trial will be modified to eliminate the safety issue. In contrast, data on other types of non-seious adverse events will be recorded but not immediately reported to the ethics committee. These data will be collected for both groups by asking participants at 6 and 26 weeks to recall any events related to their condition or the intervention.

\section{Provenance}

This trial is registered at the Australian and New Zealand Clinical trial registry. It will be conducted in accordance with the National Health and Medical Research Council's National Statement on Ethical Conduct in Human Research $^{28}$ and the Note for Good Clinical Practice (CPMP/ICH-135/95). ${ }^{29}{ }^{30}$ This trial was not commissioned and was peer reviewed for ethical and funding approval prior to submission.

\section{Trial status}

The first participant was randomised on 19 March 2019, and it is anticipated that the last participant will be recruited at the end of December 2021. Recruitment was 
stopped between March 2020 and July 2020, and severely limited until December 2020 due to the global COVID-19 pandemic. The most recent version of the protocol is V.1.2 dated November 2019.

\section{Dissemination plan}

The results of this study will be submitted for publication to peer-reviewed journals and be presented at national and international conferences.

\section{Author affiliations}

${ }^{1}$ Kolling Institute, The University of Sydney, Sydney, New South Wales, Australia

${ }^{2}$ Kolling Institute, Northern Sydney Local Health District, Sydney, New South Wales, Australia

${ }^{3}$ Department of Physiotherapy, South Western Sydney Local Health District, Sydney, New South Wales, Australia

${ }^{4}$ School of Public Health, The University of Sydney, Sydney, New South Wales, Australia

${ }^{5}$ Physiotherapy Department, Blacktown \& Mount Druitt Hospital, Sydney, New South Wales, Australia

${ }^{6}$ Department of Physiotherapy, Campbelltown Hospital, Sydney, New South Wales, Australia

${ }^{7}$ Department of Physiotherapy, Liverpool Hospital, Sydney, New South Wales,

Australia

${ }^{8}$ Department of Physiotherapy, Bankstown Hospital, Sydney, New South Wales, Australia

${ }^{9}$ Department of Physiotherapy, Royal North Shore Hospital, Sydney, New South Wales, Australia

${ }^{10}$ Sydney School of Public Health, University of Sydney, Sydney, New South Wales, Australia

${ }^{11}$ Institute for Musculoskeletal Health, The University of Sydney, Sydney, New South Wales, Australia

${ }^{12}$ Institute of Bone and Joint Research, University of Sydney, Sydney, New South Wales, Australia

\section{Twitter Catherine Sherrington @cathiesherr}

Acknowledgements The authors acknowledge support from the health professionals, patients and staff at each of the sites.

Contributors LAH, JVG, HGW, JC, CGM, CS, MDJ, MLF and AJH were responsible for the design of the intervention and the trial. LAH, JVG, HGW, MLF, BAL, AJH, DAT, IJS and MDJ secured funding. AJH is responsible for the economics analysis. TEL, JC, HGW, AB, JJC, KD and BAP are responsible for collecting data. LS, JJC, IJS, BAP $\mathrm{DW}, \mathrm{KD}, \mathrm{AB}, \mathrm{MJT}$, MDJ and HGW are responsible for the sites. All authors have read and approved the final manuscript.

Funding This project has received funding through Australia's Medical Research Future Fund Rapid Applied Research Translation Program grant awarded to Sydney Health Partners.

Competing interests None declared.

Patient consent for publication Not required.

Provenance and peer review Not commissioned; externally peer reviewed.

Supplemental material This content has been supplied by the author(s). It has not been vetted by BMJ Publishing Group Limited (BMJ) and may not have been peer-reviewed. Any opinions or recommendations discussed are solely those of the author(s) and are not endorsed by BMJ. BMJ disclaims all liability and responsibility arising from any reliance placed on the content. Where the content includes any translated material, BMJ does not warrant the accuracy and reliability of the translations (including but not limited to local regulations, clinical guidelines, terminology, drug names and drug dosages), and is not responsible for any error and/or omissions arising from translation and adaptation or otherwise.

Open access This is an open access article distributed in accordance with the Creative Commons Attribution Non Commercial (CC BY-NC 4.0) license, which permits others to distribute, remix, adapt, build upon this work non-commercially, and license their derivative works on different terms, provided the original work is properly cited, appropriate credit is given, any changes made indicated, and the use is non-commercial. See: http://creativecommons.org/licenses/by-nc/4.0/.
ORCID iDs

Hannah G Withers http://orcid.org/0000-0001-5319-7757

Catherine Sherrington http://orcid.org/0000-0001-8934-4368

\section{REFERENCES}

$1 \mathrm{Kyu} \mathrm{HH}$, Abate D, Abate KH, et al. Global, regional, and national disability-adjusted life-years (DALYs) for 359 diseases and injuries and healthy life expectancy (HALE) for 195 countries and territories, 1990-2017: a systematic analysis for the global burden of disease study 2017. Lancet 2018;392:1859-922.

2 Australian Institute of Health and Welfare. Australia's health series no. 15. Cat. no. AUS 199. Canberra: AlHW, 2016.

3 Australian Institute of Health and Welfare. Musculoskeletal conditions and comorbidity in Australia. Arthritis series no. 25. Cat. no. PHE 241 Canberra: AlHW, 2019

4 Briggs AM, Woolf AD, Dreinhöfer K, et al. Reducing the global burden of musculoskeletal conditions. Bull World Health Organ 2018;96:366-8.

5 Murray CJL, Lopez AD. Measuring the global burden of disease. $N$ Engl J Med 2013;369:448-57.

6 Arroll B, Robb G, Kool B, et al. Diagnosis and management of soft tissue shoulder injuries and related disorders. New Zealand: New Zealand Accident Compensation Corporation (ACC) and The New Zealand Guidelines Group (NZGG), 2004.

7 Motor Accidents Authority. Guidelines for the management of acute whiplash associated disorders for health professionals. Third edition. Sydney, 2014.

8 Granviken F, Vasseljen O. Home exercises and supervised exercises are similarly effective for people with subacromial impingement: a randomised trial. J Physiother 2015;61:135-41.

9 Green S, Buchbinder R, Hetrick SE, et al. Physiotherapy interventions for shoulder pain. Cochrane Database Syst Rev 2003;30.

10 Coppola SM, Collins SM. Is physical therapy more beneficial than unsupervised home exercise in treatment of post surgical knee disorders? A systematic review. Knee 2009;16:171-5.

11 Feger MA, Herb CC, Fraser JJ, et al. Supervised rehabilitation versus home exercise in the treatment of acute ankle sprains: a systematic review. Clin Sports Med 2015;34:329-46.

12 Fischer DA, Tewes DP, Boyd JL, et al. Home based rehabilitation for anterior cruciate ligament reconstruction. Clin Orthop Relat Res 1998:194-9.

13 Han ASY, Nairn L, Harmer AR, et al. Early rehabilitation after total knee replacement surgery: a multicenter, noninferiority, randomized clinical trial comparing a home exercise program with usual outpatient care. Arthritis Care Res 2015;67:196-202.

14 Kuhn JE. Exercise in the treatment of rotator cuff impingement: a systematic review and a synthesized evidence-based rehabilitation protocol. J Shoulder Elbow Surg 2009;18:138-60.

15 Odole AC, Ojo OD. A Telephone-based physiotherapy intervention for patients with osteoarthritis of the knee. Int $J$ Telerehabil 2013:5:11-20.

16 Rajan RA, Pack Y, Jackson $\mathrm{H}$, et al. No need for outpatient physiotherapy following total knee arthroplasty: a randomized trial of 120 patients. Acta Orthop Scand 2004;75:71-3.

17 Şenbursa G, Baltaci G, Atay Ö Ahmet. The effectiveness of manual therapy in supraspinatus tendinopathy. Acta Orthop Traumatol Turc 2011;45:162-7.

18 Stratford P, Gill C, Westaway M. Assessing disability and change on individual patients: a report of a patient specific measure. Physiotherapy Canada 1995;47:258-63.

19 Wong B, Ward D, Gemmell K, et al. How is telehealth being utilized in the context of rehabilitation for lower limb musculoskeletal disorders: a scoping review. Physical Therapy Rev 2020;25:350-60.

20 Cottrell MA, Russell TG. Telehealth for musculoskeletal physiotherapy. Musculoskelet Sci Pract 2020;48:102193.

21 Horn KK, Jennings S, Richardson G, et al. The patient-specific functional scale: psychometrics, clinimetrics, and application as a clinical outcome measure. J Orthop Sports Phys Ther 2012;42:30-D17.

22 Chow CK, Redfern J, Hillis GS, et al. Effect of lifestyle-focused text messaging on risk factor modification in patients with coronary heart disease: a randomized clinical trial. JAMA 2015;314:1255-63.

23 Cleland JA, Fritz JM, Childs JD. Psychometric properties of the fearavoidance beliefs questionnaire and Tampa scale of Kinesiophobia in patients with neck pain. Am J Phys Med Rehabil 2008;87:109-17.

24 Bijur PE, Silver W, Gallagher EJ. Reliability of the visual analog scale for measurement of acute pain. Acad Emerg Med 2001;8:1153-7.

25 Sayers SP, Jette AM, Haley SM, et al. Validation of the late-life function and disability instrument. J Am Geriatr Soc 2004;52:1554-9. 
26 Late-life function \& disability instrument. Available: https:// geriatrictoolkit.missouri.edu/funct/lfdi.htm [Accessed 05/05/2021].

27 Kenward MG, Carpenter J. Multiple imputation: current perspectives. Stat Methods Med Res 2007;16:199-218.

28 National Health and Medical Research Council. Guidance: safety monitoring and reporting in clinical trials involving therapeutic goods. Canberra: National Health and Medical Research Council, 2016.
29 The Therapeutic Goods Administration. CPMP/ICH note for guidance on good clinical practice (CPMP/ICH-135/95. Canberra: Commonwealth of Australia, 2000.

30 International Council for Harmonisation of technical requirements for pharmaceuticals for human use (ICH). ICH harmonised guideline: integrated addendum to ICH E6(r1): guideline For good clinical practice, 2016. 